\title{
Non-linear associations of body mass index with impaired fasting glucose, $\beta$-cell dysfunction, and insulin resistance in nondiabetic Chinese individuals: a cross-sectional study
}

\author{
Min Chen ${ }^{\circledR 1}{ }^{1,2}$, Ruihua Yang ${ }^{\left({ }^{3} 3\right.}$, Ying Wang ${ }^{(1)}$, Yumei Jia ${ }^{\circledR 1}$, Jia Liu ${ }^{\circledR 1}$, Guang Wang ${ }^{(1)} 1$ \\ ${ }^{1}$ Department of Endocrinology, Beijing Chao-Yang Hospital, Capital Medical University, Beijing, China \\ ${ }^{2}$ Department of Endocrinology, Fu Xing Hospital, Capital Medical University, Beijing, China \\ ${ }^{3}$ Department of Endocrinology, Beijing First Hospital of Integrated Chinese and Western Medicine, Beijing, China \\ ${ }^{4}$ The Physical Examination Centre, Beijing Chao-Yang Hospital, Capital Medical University, Beijing, China
}

\begin{abstract}
Introduction: Identifying and managing patients with prediabetes is important. The study aims to investigate the association of body mass index (BMI) with impaired fasting glucose (IFG), $\beta$-cell dysfunction, and insulin resistance in nondiabetic Chinese individuals. Material and methods: This was a cross-sectional study of consecutive nondiabetic individuals enrolled between January 2014 and January 2015, divided into NFG [normal fasting glucose, fasting blood glucose (FBG) $<5.6 \mathrm{mmol} / \mathrm{L}$ ) and IFG $(\mathrm{n}=450 ; \mathrm{FBG} \geq 5.6 \mathrm{mmol} / \mathrm{L})$ groups. Restricted cubic splines and piecewise-regression were used to model the association of IFG, impaired $\beta$-cell function, and insulin resistance with BMI. Stratified analyses were performed across sex and age.

Results: A total of 900 NFG and 450 IFG individuals were enrolled, with a median age of 41 (30-49) years and 1076 males (79.7\%). After adjusting for age and sex, the restricted cubic splines showed that the risk of IFG was increasing rapidly until around $27.96 \mathrm{~kg} / \mathrm{m}^{2}$ of BMI and then started to plateau afterward ( $p$ for non-linearity $=0.010$ ), which was similar in males and individuals $\leq 45$ years old ( $p$ for non-linearity $<0.001$ and $=0.007$, respectively). The risk of insulin resistance increased and $\beta$-cell dysfunction decreased as the BMI increased in all participants (both $\mathrm{p}$ for non-linearity $>0.05$ ), consistent with the results in males, females, and $\leq 45$ and $>45$ year olds.

Conclusions: The risk of IFG does not rise linearly as the BMI increases, and higher BMI seems to decelerate the rise of the risk. (Endokrynol Pol 2021; 72 (6): 618-624)
\end{abstract}

Key words: insulin-secreting cells; insulin resistance; blood glucose; body mass index

\section{Introduction}

The prevalence of diabetes in Chinese adults has been increasing rapidly since 2000, with an annual growth rate of $0.72 \%$ (95\% CI: $0.34-1.10 \%$ ) [1]. In a nationally representative cross-sectional survey carried out in 2013, the estimated overall prevalence of diabetes among adults in China was $10.9 \%$, and that for prediabetes was $35.7 \%$ [2]. Once developed, type 2 diabetes (T2DM) is an irreversible condition [3]. Individuals with prediabetes will eventually progress to overt diabetes, but it can be delayed with the appropriate changes in lifestyle habits and with drugs when necessary. Individuals with prediabetes will have no signs or symptoms but will show impaired fasting glucose (IFG). Identifying and managing patients with prediabetes is important because chronic hyperglycaemia of diabetes can lead to multiorgan damage resulting in renal, neurological, cardiovascular, and other serious complications [3] and is associated with significant morbidity and mortality [4].

The major underlying pathophysiology of T2DM in the Chinese population is the same as in other populations, i.e. $\beta$-cell dysfunction and insulin resistance [5]. Insulin resistance is defined as an impaired peripheral sensitivity to normal or elevated insulin levels. Compensatory hyperinsulinaemia occurs when insulin secretion increases to maintain blood glucose levels at normal levels. Hence, high fasting insulin (FINS) levels may indicate insulin resistance [6]. The abnormal function of pancreatic islet $\beta$-cells is characterized by impairments ranging from impaired insulin pulse secretion to the lack of insulin secretion in the first phase and increased compensation in the second phase [7]. In the decompensated stage, both the 1- and 2-phase secretions are decreased. In the United Kingdom Pro- 
spective Diabetes Study (UKPDS), the $\beta$-cell function was lower by about $50 \%$ when fasting hyperglycaemia was diagnosed [8].

High adiposity, as reflected by a high body mass in$\operatorname{dex}(\mathrm{BMI})$, is the most important independent risk factor for T2DM [9]. Homeostasis model assessment (HOMA) equations are tools used to estimate insulin resistance. HOMA-IR is used to assess insulin resistance, while HOMA- $\beta$ is used to assess pancreatic $\beta$-cell function [10]. A study in Caucasians showed that the HOMA-IR is dependent upon sex, age, and BMI categories, with obese people having high HOMA-IR [11]. Both $\beta$-cell dysfunction and insulin resistance contribute to high levels of fasting blood glucose (FBG) and the progressive deterioration from impaired glucose regulation (IGR) to T2DM; when IGR signs start to manifest, FBG may still be in the normal range [12]. The degree of $\beta$-cell dysfunction is a determinant for the FBG levels, with decreased insulin secretion by $\beta$-cells and peripheral insulin resistance contributing to increased FBG levels [13]. In subjects with T2DM, $\beta$-cell secretory capacity is reduced by approximately $75 \%$ when the FBG levels increase [14]. Previous studies have suggested that deterioration of basal and early-phase insulin secretion, rather than insulin sensitivity, is crucial to the progression from normal glucose tolerance (NGT) to T2DM [15]

Nevertheless, there is a lack of data about the relationship between FBG and $\beta$-cell function during the progression from NFG to IFG according to the different BMI levels. Therefore, this study aimed to investigate the association of BMI with IFG, $\beta$-cell dysfunction, and insulin resistance in nondiabetic Chinese individuals. The results could help identify the individuals at higher risk of progression to T2DM in whom early lifestyle changes should be undertaken.

\section{Material and methods}

\section{Study design and participants}

This cross-sectional study enrolled consecutive nondiabetic individuals from the Physical Examination Centre of our Hospital from January 2014 to January 2015. This study was approved by the Ethics Committee of our Hospital. All participants provided written informed consent.

The inclusion criteria were as follows: 1) 18-80 years of age; 2) no medical history of heart disease, brain disease, lung disease, high blood pressure (systolic blood pressure (SBP) $\geq 140 \mathrm{mmHg}$ and/or diastolic blood pressure (DBP) $\geq 90 \mathrm{mmHg}$ measured 3 times on different days, without medication), blood lipid disorders (total cholesterol (TC) $\geq 5.2 \mathrm{mmol} / \mathrm{L}$, low-density lipoprotein cholesterol $(\mathrm{LDL}-\mathrm{C}) \geq 3.4 \mathrm{mmol} / \mathrm{L}$, triglyceride $(\mathrm{TG}) \geq 1.7 \mathrm{mmol} / \mathrm{L}$, or highdensity lipoprotein cholesterol (HDL-C) $<1.0 \mathrm{mmol} / \mathrm{L}$, without medication), chronic hepatitis (viral hepatitis, autoimmune liver disease, drug-induced liver disease, liver cancer and cirrhosis, and no history of medication), and chronic kidney disease (chronic glomerulopathy, renal tubular disease, interstitial nephritis, and renal vascular disease, and the serum creatinine is normal), and 3 ) markers of liver and kidney functions were all within the normal range. The exclusion criteria were 1) $\mathrm{FBG} \geq 7.0 \mathrm{mmol} / \mathrm{L}$ or glycated hemoglobin $\left.\left(\mathrm{HbA}_{1}\right) \geq 6.5 \%, 2\right)$ use of anti-hypertension drugs, anti-dyslipidemia drugs, or any medications known to affect insulin sensitivity within the past 6 months, including weight-loss drugs, Chinese herbal medicines, anti-diabetic drugs, and insulin; 3) severe organ dysfunction or mental illness; or 4) history of diabetes mellitus, severe anaemia, pancreatitis, acute myocardial infarction, or stroke.

\section{Grouping}

According to the diagnostic criteria for IFG by the American Diabetes Association (ADA) in the United States [16], the individuals were divided into 2 groups based on FBG: IFG (FBG $\geq 5.6 \mathrm{mmol} / \mathrm{L}$ ) and NFG $($ FBG $<5.6 \mathrm{mmol} / \mathrm{L})$

\section{Data collection and definitions}

Basic demographic data (i.e. age, sex, body height, and weight) were collected. Height and weight were measured to the nearest $0.1 \mathrm{~cm}$ and $0.1 \mathrm{~kg}$ by the same well-trained examiner. Body mass index (BMI) was calculated as follows:

\section{body weight $(\mathrm{kg}) /[\text { height }(\mathrm{m})]^{2}$}

Fasting (overnight) serum samples were collected from a peripheral vein. Serum samples were stored at $-80^{\circ} \mathrm{C}$. TC, HDL-C, LDL-C, and TG levels were measured by colorimetric enzymatic assays using a 7170 autoanalyzer (Hitachi, Tokyo, Japan). Reference intervals for TC, HDL-C, LDL-C, and TG were $3.62-5.70 \mathrm{mmol} / \mathrm{L}$, $1.03-1.55 \mathrm{mmol} / \mathrm{L}, 1.81-3.36 \mathrm{mmol} / \mathrm{L}$, and $0.56-1.70 \mathrm{mmol} / \mathrm{L}$, respectively [17]. $\mathrm{HbA}_{1 c}$ was measured using a Variant II $\mathrm{HbA}_{1 c}$ analyser (Bio-Rad Laboratories, Hercules, CA, USA). Alanine aminotransferase (ALT), aspartate aminotransferase (AST), FBG, $\mathrm{HbA}$, and FINS levels were measured routinely at the central biochemistry laboratory of our hospital.

The HOMA-IR and HOMA- $\beta$ were determined. HOMA-IR was calculated according to the following formula:

$$
H O M A-I R=[F B G(m m o l / L) \times F I N S(\mu I U / m L)] / 22.5,
$$

And HOMA- $\beta$ was calculated as follows:

$$
H O M A-\beta=[20 \times \text { FINS }(\mu I \mathrm{~L} / \mathrm{mL})] /[F B G(\mathrm{mmol} / \mathrm{L})-3.5] .
$$

Insulin resistance was defined as higher than the $75^{\text {th }}$ percentile of HOMA-IR in participants with a normal BMI and normal fasting glucose [18], which was 2.726 in this study. Impaired $\beta$-cell function was defined as lower than the $25^{\text {th }}$ percentile of the HOMA- $\beta$ in participants with a normal BMI and normal fasting glucose [12, 18], which was 73.988 in this study.

\section{Statistical analysis}

Continuous data were presented as mean \pm standard deviation or median (upper and lower quartiles) and analysed using Student's t-test. Categorical variables are presented as frequencies and were analysed using the chi-square test. The significance of the mean differences for the indexes of insulin resistance and $\beta$-cell function (HOMA-IR, HOMA- $\beta$, and FINS) was tested using general linear models after adjustment for age, sex, and BMI. The associations between insulin resistance, $\beta$-cell dysfunction, and IFG with BMI were evaluated by restricted cubic spline regression, adjusting for age and sex. The number of dots was chosen to 5 knots at the $5^{\text {th }}$ $35^{\text {th }}, 65^{\text {th }}$, and $95^{\text {th }}$ centiles of BMI. Piecewise-regression models were then performed to quantify associations: where there was evidence of non-linearity, a piecewise-regression model with a single change point was estimated by trying all possible values for the change point and choosing the value with the highest likelihood. Moreover, stratified analyses were performed to explore whether the association varied across sex and age. Data analysis was per- 
formed using SPSS 22.0 (IBM, Armonk, NY, USA) and R 4.0.2 (The $\mathrm{R}$ Project for Statistical Computing, www.r-project.org). GraphPad Prism 8 (GraphPad Software Inc., San Diego, CA, USA) was used to draw the figures. All tests were two-tailed, and $p$ values $<0.05$ were considered statistically significant.

\section{Results}

\section{Characteristics of the participants}

A total of 1350 participants, including 900 individuals in the NFG group and 450 in the IFG group, were enrolled from the Physical Examination Centre of our Hospital between January 2014 and January 2015 and analysed. The mean age was 41 (30-49) years and most participants were male $(79.6 \%)$ (Tab. 1). There were significant differences in age, BMI, and blood pressure between the two groups (all $\mathrm{p}<0.05$ ).

In addition, higher levels of TC, TG, and LDL-C and lower levels of HDL-C were observed in the IFG group (all $\mathrm{p}<0.05$ ). Compared with the NFG group, FINS and HOMA-IR were significantly higher in the IFG group (FINS: $12.75 \pm 0.24$ vs. $15.35 \pm 0.34$; HOMA-IR: $2.97 \pm 0.06$ vs. $4.09 \pm 0.09)$, while HOMA- $\beta$ was lower $(150.1 \pm 2.4$ vs. $125.6 \pm 3.5)($ all $\mathrm{p}<0.05)$.

\section{Association of IFG, impaired $\beta$-cell function, and insulin resistance with $B M I$}

After adjusting for age and sex, restricted cubic spline regression showed that the risk of insulin resistance increased rapidly as the BMI increased, and the non-linearity was not significant ( $p=0.725$, Fig. 1A), indicating that the risk of insulin resistance increased as BMI increased, or, in some way, high BMI was a risk factor of insulin resistance. In contrast, the risk of $\beta$-cell dysfunction decreased in an approximately linear fashion ( $\mathrm{p}$ for non-linearity $=0.851$, Fig. $1 \mathrm{~B}$ ), meaning that the risk of $\beta$ cell dysfunction decreased as BMI increased, or, in some way, high BMI was a protective factor of $\beta$-cell function. Notably, the risk of IFG increased rapidly until around $27.96 \mathrm{~kg} / \mathrm{m}^{2}$ of BMI and then started to plateau afterward ( $\mathrm{p}$ for non-linearity $=0.010$, Fig. $1 \mathrm{C}$ ), indicating that the risk of IFG increased as the BMI increased, but higher $\mathrm{BMI}$ seemed to decelerate the increase of the risk.

\section{Association of IFG, impaired $\beta$-cell function, and insulin resistance with BMI by age}

After stratifying all participants into the 18-45and $>45$-year-old subgroups, younger individuals

Table 1. Characteristics of the subjects

\begin{tabular}{|c|c|c|c|c|}
\hline Characteristics & $\begin{array}{c}\text { Total } \\
\text { (n = 1350) }\end{array}$ & $\begin{array}{c}\text { NFG } \\
(n=900)\end{array}$ & $\begin{array}{c}\text { IFG } \\
(n=450)\end{array}$ & p \\
\hline Age [years] & $41(30,49)$ & $37(29,47)$ & $45(37,52)$ & $<0.001$ \\
\hline Males [n (\%)] & $1076(79.7)$ & $682(75.8)$ & $394(87.6)$ & $<0.001$ \\
\hline $\mathrm{BMI}\left[\mathrm{kg} / \mathrm{m}^{2}\right]$ & $24.8(22.3,27.0)$ & $24.3(21.7,26.7)$ & $25.8(23.9,27.7)$ & $<0.001$ \\
\hline SBP [mmHg] & $123(114,133)$ & $121(112,130)$ & $127(118,136)$ & $<0.001$ \\
\hline $\mathrm{DBP}[\mathrm{mmHg}]$ & $74(68,82)$ & $73(67,80)$ & $79(72,86)$ & $<0.001$ \\
\hline ALT [U/L] & $23(16,34)$ & $21(16,32)$ & $26(19,37)$ & $<0.001$ \\
\hline AST [U/L] & $20(17,24)$ & $19(16,23)$ & $21(18,25)$ & $<0.001$ \\
\hline TG [mmol/L] & $1.25(0.83,1.93)$ & $1.12(0.77,1.77)$ & $1.51(0.98,2.26)$ & $<0.001$ \\
\hline TC [mmol/L] & $4.89(4.33,5.54)$ & $4.81(4.22,5.43)$ & $5.07(4.55,5.74)$ & $<0.001$ \\
\hline HDL-C [mmol/L] & $1.19(1.03,1.41)$ & $1.21(1.04,1.44)$ & $1.15(1.00,1.35)$ & $<0.001$ \\
\hline LDL-C [mmol/L] & $2.83(2.38,3.36)$ & $2.76(2.32,3.33)$ & $2.94(2.55,3.39)$ & $<0.001$ \\
\hline $\mathrm{FBG}[\mathrm{mmol} / \mathrm{L}]$ & $5.40(5.13,5.71)$ & $5.23(5.03,5.40)$ & $5.88(5.71,6.15)$ & $<0.001$ \\
\hline $\mathrm{HBA}_{1 \mathrm{c}}(\%)$ & $5.7(5.5,5.9)$ & $5.5(5.4,5.7)$ & $5.8(5.6,6.0)$ & $<0.001$ \\
\hline FINS [mU/L] & $11.71(8.08,16.60)$ & $10.67(7.38,15.46)$ & $13.41(9.72,18.72)$ & $<0.001$ \\
\hline FINS $[\mathrm{mU} / \mathrm{L}]^{\mathrm{a}}$ & $14.05 \pm 0.20$ & $12.75 \pm 0.24$ & $15.35 \pm 0.34$ & $<0.001$ \\
\hline HOMA-IR & $2.86(1.90,4.07)$ & $2.51(1.69,3.63)$ & $3.55(2.62,4.97)$ & $<0.001$ \\
\hline HOMA-IR & $3.53 \pm 0.05$ & $2.97 \pm 0.06$ & $4.09 \pm 0.09$ & $<0.001$ \\
\hline HOMA- $\beta$ & $120.0(84.5,172.6)$ & $126.5(88.0,181.2)$ & $111.5(78.2,156.1)$ & $<0.001$ \\
\hline HOMA- $\beta^{\text {a }}$ & $137.9 \pm 2.1$ & $150.1 \pm 2.4$ & $125.6 \pm 3.5$ & $<0.001$ \\
\hline
\end{tabular}

Normally distributed variables are expressed as mean \pm standard deviation (SD), while variables with skewed distribution (age, ALT, AST, TG, FINS, HOMA-IR, and HOMA- $\beta$ are expressed as medians [upper and lower quartiles]). BMI: — body mass index; SBP: — systolic blood pressure; DBP: — diastolic blood pressure; ALT: - alanine aminotransferase; AST: — aspartate aminotransferase; TC: — total cholesterol; TG: - triglyceride; HDL-C: — high-density lipoprotein cholesterol; LDL-C: - low-density lipoprotein cholesterol; FBG: — fasting blood glucose; FINS: — fasting insulin; HOMA-IR: - homeostasis model assessment of insulin resistance; HOMA- $\beta$ : - homeostasis model assessment of $\beta$-cell function; adjusted for age, sex, and BMI 


\section{A}

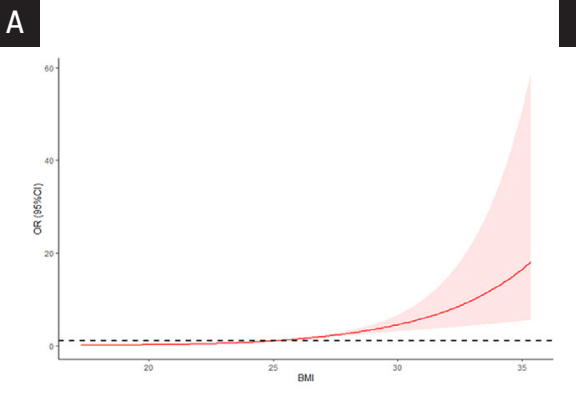

B

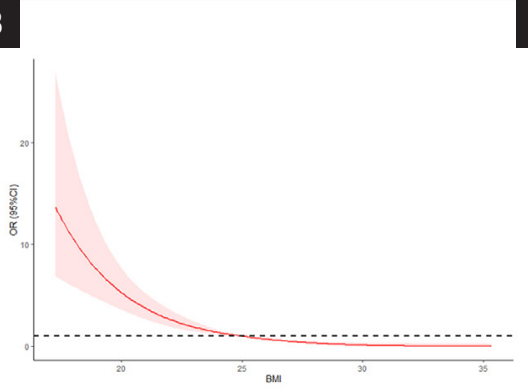

C

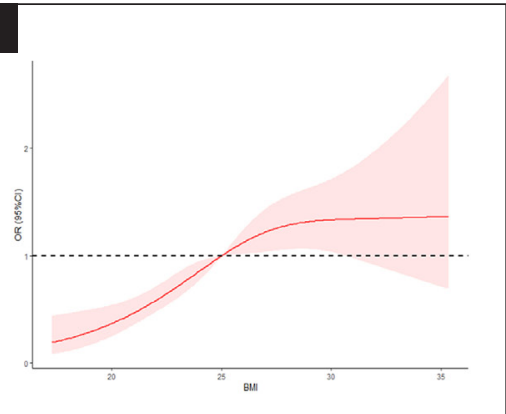

Figure 1. Association between body mass index and insulin resistance (A), $\beta$-cell dysfunction (B), and impaired fasting glucose (IFG) (C)

\section{A}

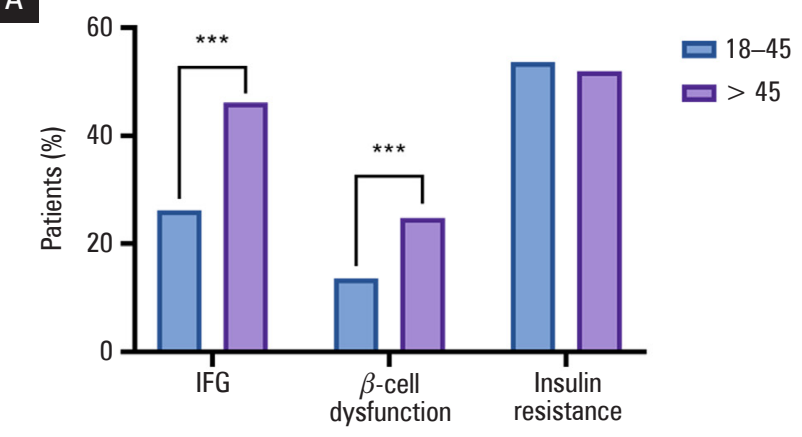

B

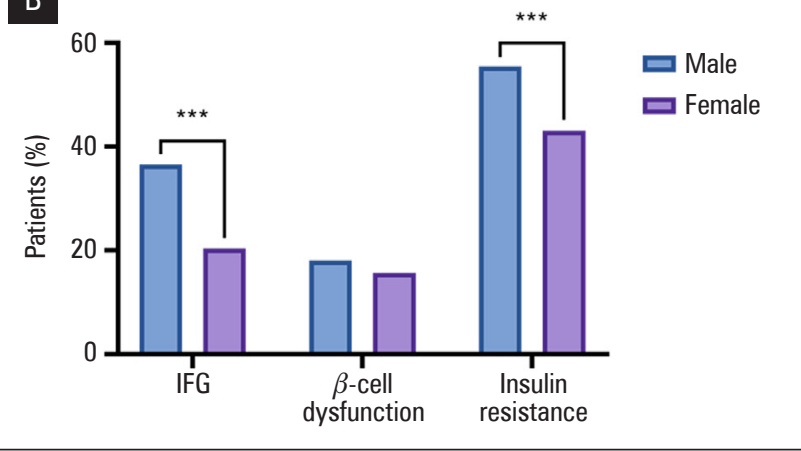

Figure 2. Prevalence of impaired fasting glucose (IFG), $\beta$-cell dysfunction, and insulin resistance among nondiabetic patients by age (A) and sex (B). ${ }^{* *}$ represented $p<0.001$

showed a lower prevalence of IFG and $\beta$-cell dysfunction (both $\mathrm{p}<0.001$, Fig. 2A). Meanwhile, no significant difference was found regarding the prevalence of insulin resistance. Restricted cubic spline regression showed that the risk of insulin resistance increased and $\beta$-cell dysfunction decreased as BMI increased, and the non-linearity was not significant whether in younger or older individuals (all $\mathrm{p}$ for non-linearity $>0.05$, Fig. 3A, B, D, and E). Consistent with asll of the individuals, the risk of IFG increased rapidly until around $27.51 \mathrm{~kg} / \mathrm{m}^{2}$ of BMI and then started to plateau afterward in 18-45-year-old individuals ( $\mathrm{p}$ for non-linearity $=0.007$, Fig. $3 \mathrm{C}$ ). On the other hand, the non-linearity seemed to disappear in older individuals ( $p$ for non-linearity $=0.212$, Fig. 3E). The results suggest that in younger individuals, the risk of IFG increased as the BMI increased, but higher BMI seemed to decelerate the increase of the risk, while still being linked to increased risk in older individuals.

\section{Association of IFG, impaired $\beta$-cell function, and insulin resistance with BMI by sex}

Stratified analysis showed that male individuals had a higher prevalence of IFG and insulin resistance (both $\mathrm{p}<0.001$, Fig. 2B). Meanwhile, no significant difference was found regarding the prevalence of $\beta$-cell dysfunc- tion. Restricted cubic spline regression showed that the risk of insulin resistance increased and impaired $\beta$-cell function decreased as BMI increased, and the non-linearity was not significant whether in male or female individuals (all $\mathrm{p}$ for non-linearity $>0.05$, Fig. 4A, B, D, and E). As with all the individuals, the risk of IFG increased rapidly until around $24.26 \mathrm{~kg} / \mathrm{m}^{2}$ of BMI and then started to plateau afterward in male individuals ( $\mathrm{P}$ for non-linearity $<0.001$, Fig. 4C). However, the non-linearity seemed to disappear in female individuals ( $\mathrm{p}$ for non-linearity $=0.349$, Fig. $4 \mathrm{E})$. Hence, in males, the risk of IFG increased as the $\mathrm{BMI}$ increased, but higher BMI seemed to decelerate the increase of the risk, while high BMI still increased the risk in females.

\section{Discussion}

This study aimed to investigate the association of BMI with IFG, $\beta$-cell dysfunction, and insulin resistance in nondiabetic Chinese individuals. The results show that the risk of IFG is not rising linearly as the BMI increases, and higher BMI seems to decelerate the rise of the risk. The findings suggest that the compensated secretion of $\beta$ cells might play a valuable role against insulin resistance in high-BMI individuals. 


\section{A}

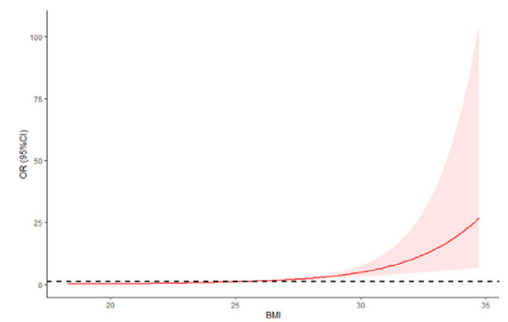

D

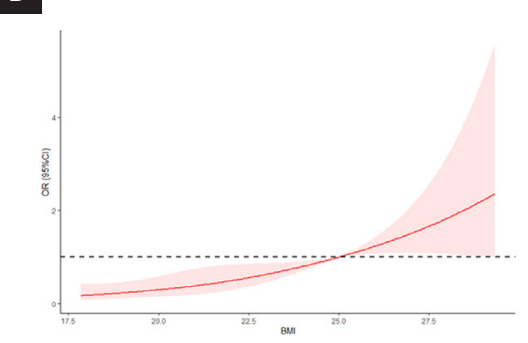

B

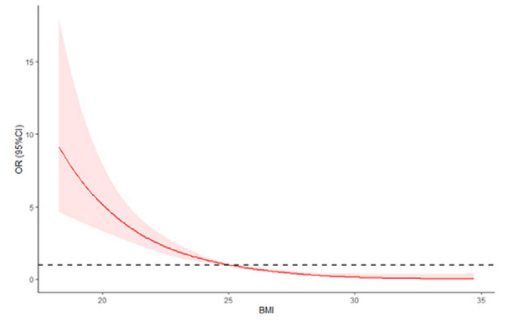

E

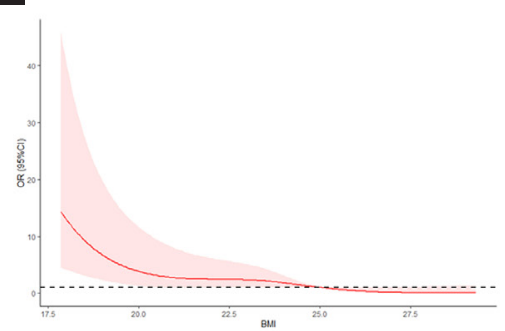

c

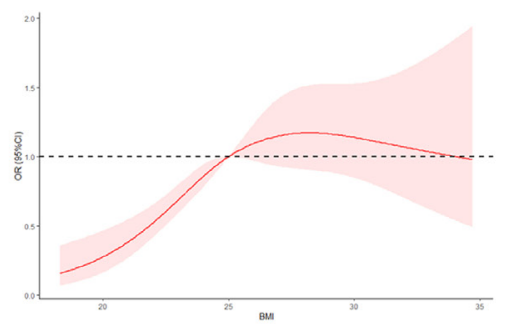

$\mathrm{F}$

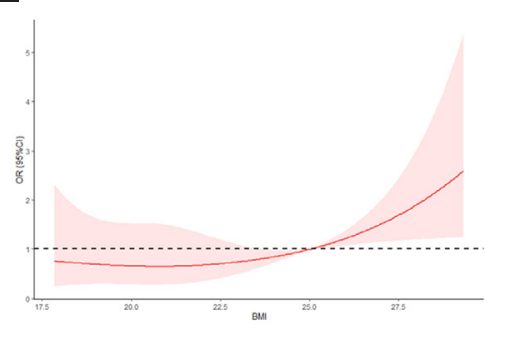

Figure 3. Association between body mass index and insulin resistance (A, D), $\beta$-cell dysfunction (B, E), and impaired fasting glucose (IFG) (C, F) in nondiabetic 18-45-(A-C) and > 45-year-old (D-E) individuals

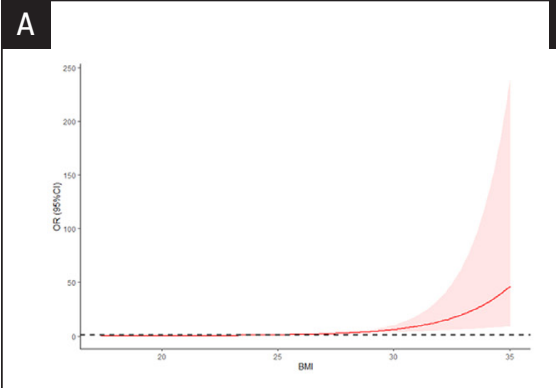

D

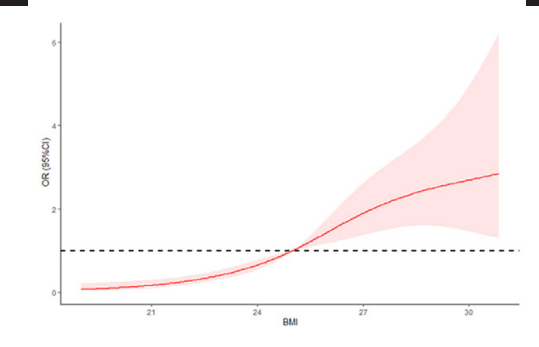

B

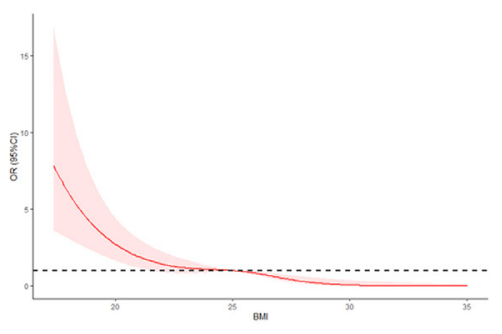

E

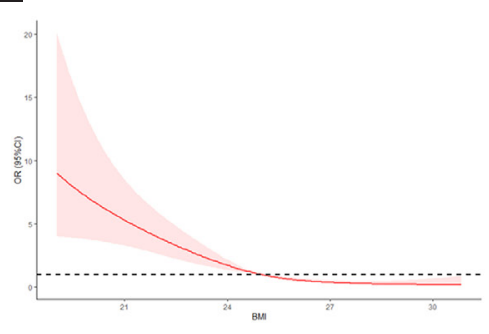

c

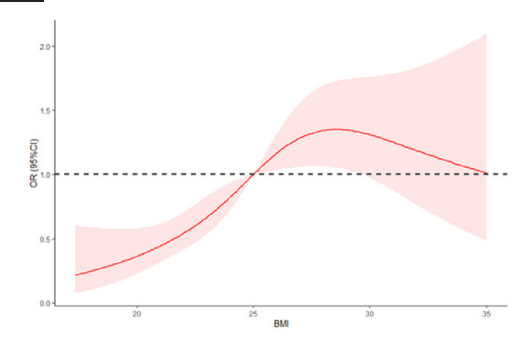

$\mathrm{F}$

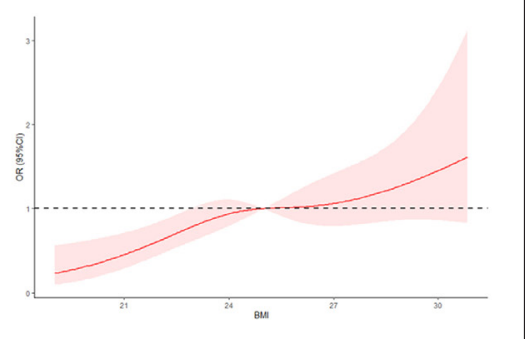

Figure 4. Association between body mass index and insulin resistance (A, D), $\beta$-cell dysfunction (B, E), and impaired fasting glucose (IFG) $(\mathbf{C}, \mathbf{F})$ in nondiabetic male (A-C) and female (D-E) individuals

Overweight or obesity is a major risk factor for prediabetes and T2DM. In Northeast China, the prevalence of overweight is $42 \%$, the prevalence of obesity is $20.1 \%$, and the prevalence of central obesity is $58.9 \%$ in adults with prediabetes [19]. Abdominal obesity accounts for $28.1 \%$ of incident cases of diabetes among men and $41.2 \%$ among women [20]. FINS and HOMA-IR were higher in the IFG group than in the NFG group, while HOMA- $\beta$ was lower in the IFG group. It is consistent with the concept of progressive insulin resistance and $\beta$-cell dysfunction from NFG to T2DM [14]. High FBG is one of the main characteristics of T2DM. In the postprandial state, T2DM defined by $\mathrm{HbA}_{1 \mathrm{c}}$ levels was associated with both $\alpha$ - and $\beta$-cell dysfunction and impaired insulin response combined with non-suppressed glucagon [21]. In the fasting state, the liver maintains blood glucose levels for the brain's need for glucose. Thus, insulin resistance of the liver plays a key role in 
high FBG levels. IFG is an intermediate status between NFG and T2DM, and the present study showed that HOMA-IR increased from NFG to IFG, which is supported by the literature $[11,22]$.

In addition to insulin resistance, $\beta$-cell dysfunction is well recognized as central to the pathophysiology of prediabetes and diabetes. The earliest detectable abnormality in individuals at risk of T2DM is insulin hypersecretion, which aims to compensate for insulin resistance in peripheral tissues. Nevertheless, T2DM does not occur until the $\beta$-cells become unable to secrete enough insulin to overcome peripheral insulin resistance. In some Chinese analyses evaluating the role of $\beta$-cell function in increasing FBG, the disposition index, a value representing $\beta$-cell function, was decreased by $38 \%$ in IFG [12], and HOMA- $\beta$ progressively declined by $33 \%$ in IFG and reached a substantial decrease of $41 \%$ in the diabetic range of FBG [23]. Previous studies suggested that the deterioration of basal and early-phase insulin secretion, rather than insulin sensitivity, is crucial to the progression from NGT to T2DM [15]. Sun et al. [24] found that in overweight Chinese adolescents with normal glucose tolerance, insulin resistance progressively increased with increasing BMI, but the compensatory increase in early insulin secretion was limited. Roth et al. [25]. found that the OGTT $30-\mathrm{min} / 120-\mathrm{min}$ insulin ratio was significantly lower in obese children, which indicated that these children already had inadequate $\beta$-cell compensation for the degree of insulin resistance [25]. In the present study, the cubic spline analyses showed that the risk of insulin resistance increased as the BMI increased, meaning that high BMI was a risk factor of insulin resistance. In addition, the risk of impaired $\beta$-cell function decreased as the BMI increased, suggesting that high BMI was a protective factor of impaired $\beta$-cell function. The compensated secretion of $\beta$ cells might contribute to the phenomenon.

Interestingly, different relationships were observed between younger and older individuals and between males and females. The differences of IFG and $\beta$-cell function in younger and older individuals revealed that the main cause of the higher prevalence of IFG in older individuals might be worse $\beta$-cell function. The disappearance of non-linearity in older individuals might be due to a worse $\beta$-cell function and worse compensated secretion function of $\beta$-cells. It is consistent with the literature showing that $\beta$-cell function decreases with age $[26,27]$. The differences of IFG and insulin resistance in male and female individuals revealed that the main cause of the higher prevalence of IFG in male individuals might be insulin resistance. In addition, the change point of BMI was lower in males than in whole individuals. Because of the high prevalence of insulin resistance in men, compensatory insulin secretion might be more common in men [28]. Sex hormones play a role in developing insulin resistance and T2DM $[28,29]$.

It is now recognized that $\beta$-cell failure occurs much earlier and is more severe than previously thought [30]. Individuals with FBG in the upper range have decreased $\beta$-cell function and decreased insulin sensitivity before the onset of diabetes, and Asians are more likely to have lower $\beta$-cell mass and insulin secretory capacity compared with Caucasians [31], suggesting that a small decline in $\beta$-cell function could be enough to precipitate progression to overt T2DM in Asians. A study of 1835 Japanese patients demonstrated that insulin secretory defect and decreased insulin sensitivity were found in patients with IFG in the range 5.6-6.1 [32]. The present study showed that insulin resistance and $\beta$-cell dysfunction were deteriorated when FBG was $\geq 5.6 \mathrm{mmol} / \mathrm{L}$. It may suggest that the optimal time to intervene is before the FBG is $\geq 5.6 \mathrm{mmol} / \mathrm{L}$ in the Chinese population, to prevent the progression of T2DM and its complications. It is supported by a previous study that showed distinct lipid patterns across FBG levels in different ethnicities [33].

The present study has some limitations. Firstly, we used the HOMA- $\beta$ to evaluate pancreatic $\beta$-cell function. HOMA- $\beta$ is not a precise index compared to the hyperglycaemic clamp, but the clamp is expensive and time-consuming, and it is difficult to carry out in the setting of a large-scale epidemiological study. Secondly, we did not take into account the level of physical activity of the participants, which might significantly affect FBG, insulin concentration, and insulin resistance. Finally, males outnumbered females in this study because the population were enrolled at the hospital's medical examination centre. Most participants were men with good economic conditions, which resulted in a limited analysis of female individuals. Further studies are necessary to address this issue.

\section{Conclusion}

The risk of IFG does not rise linearly as the BMI increases, and higher BMI seems to decelerate the rise of the risk. The association of BMI with the risk of IFG decelerates at high BMI values in younger individuals and males. The findings suggest that the compensated secretion of $\beta$ cells might play a valuable role against insulin resistance in high-BMI individuals. These results could help identify individuals at higher risk of progression to T2DM in whom early lifestyle changes should be undertaken, and it could contribute personalized management. 


\section{Conflict of interest}

All the authors declare no conflict of interests.

\section{Funding}

The authors acknowledge the help of the National Natural Science Foundation of China (No. 81770792).

\section{References}

1. Zhang DD, Tang X, Jin DY, et al. [Prevalence of diabetes in Chinese adults: a Meta-analysis]. Zhonghua Liu Xing Bing Xue Za Zhi. 2018; 39(6): 852-857, doi: 10.3760/cma.j.issn.0254-6450.2018.06.030, indexed in Pubmed: 29936760.

2. Wang L, Gao P, Zhang M, et al. Prevalence and Ethnic Pattern of Diabetes and Prediabetes in China in 2013. JAMA. 2017; 317(24): 2515-2523, doi: 10.1001/jama.2017.7596, indexed in Pubmed: 28655017.

3. American Diabetes Association. Diagnosis and classification of diabetes mellitus. Diabetes Care. 2014; 37 Suppl 1: S81-S90, doi: 10.2337/dc14-S081, indexed in Pubmed: 24357215.

4. Rao Kondapally Seshasai S, Kaptoge S, Thompson A, et al. Emerging Risk Factors Collaboration. Diabetes mellitus, fasting glucose, and risk of cause-specific death. N Engl J Med. 2011; 364(9): 829-841, doi: 10.1056/NEJMoa1008862, indexed in Pubmed: 21366474.

5. Ma RC, Lin Xu, Jia W. Causes of type 2 diabetes in China. Lancet Diabetes Endocrinol. 2014; 2(12): 980-991, doi: 10.1016/S2213-8587(14)70145-7, indexed in Pubmed: 25218727.

6. Wilcox G. Insulin and insulin resistance. Clin Biochem Rev . 2005; 26(2): 19-39, indexed in Pubmed: 16278749.

7. Kahn SE. Clinical review 135: The importance of beta-cell failure in the development and progression of type 2 diabetes. J Clin Endocrinol Metab. 2001; 86(9): 4047-4058, doi: 10.1210/jcem.86.9.7713, indexed in Pubmed: 11549624

8. Matthews DR, Cull CA, Stratton IM, et al. UKPDS 26: Sulphonylurea failure in non-insulin-dependent diabetic patients over six years. UK Prospective Diabetes Study (UKPDS) Group. Diabet Med. 1998; 15(4): 297-303, doi: 10.1002/(SICI)1096-9136(199804)15:4<297::A ID-DIA572>3.0.CO;2-W, indexed in Pubmed: 9585394.

9. DeFronzo RA, Ferrannini E, Groop L, et al. Type 2 diabetes mellitus. Nat Rev Dis Primers. 2015; 1: 15019, doi: 10.1038/nrdp.2015.19, indexed in Pubmed: 27189025.

10. Wallace TM, Levy JC, Matthews DR. Use and abuse of HOMA modeling. Diabetes Care. 2004; 27(6): 1487-1495, doi: 10.2337/diacare.27.6.1487, indexed in Pubmed: 15161807.

11. Chen G, Liu C, Yao J, et al. Overweight, obesity, and their associations with insulin resistance and -cell function among Chinese: a cross-sectional study in China. Metabolism. 2010; 59(12): 1823-1832, doi: 10.1016/j. metabol.2010.06.009, indexed in Pubmed: 20655552.

12. $\mathrm{Bi} Y, Z$ hu $\mathrm{D}$, Jing $Y$, et al. Decreased beta cell function and insulin sensitivity contributed to increasing fasting glucose in Chinese. Acta Diabetol. 2012; 49 Suppl 1: S51-S58, doi: 10.1007/s00592-010-0194-4, indexed in Pubmed: 20473530.

13. Cerf ME, Cerf ME, Chapman CS, et al. High-fat programming of hyperglycemia, hyperinsulinemia, insulin resistance, hyperleptinemia, and altered islet architecture in 3-month-old wistar rats. ISRN Endocrinol. 2012; 2012: 627270, doi: 10.5402/2012/627270, indexed in Pubmed: 22988521.

14. Pang C, Bao Yq, Wang C, et al. Relationship between the level of fasting plasma glucose and beta cell functions in Chinese with or without diabetes. Chin Med J. 2008; 121(21): 2119-2123, doi: 10.1097/00029330-2 00811010-00002, indexed in Pubmed: 19080169.

15. Tabák AG, Herder C, Rathmann W, et al. Prediabetes: a high-risk state for diabetes development. Lancet. 2012; 379(9833): 2279-2290, doi: 10.1016/S0140-6736(12)60283-9, indexed in Pubmed: 22683128.

16. Genuth S, Alberti KG, Bennett P, et al. Expert Committee on the Diagnosis and Classification of Diabetes Mellitus. Follow-up report on the diagnosis of diabetes mellitus. Diabetes Care. 2003; 26(11): 3160-3167, doi: 10.2337/diacare.26.11.3160, indexed in Pubmed: 14578255

17. Joint committee for guideline revision. 2016 Chinese guidelines for the management of dyslipidemia in adults. J Geriatr Cardiol. 2018, 15(1): 1-29, doi: 10.11909/j.issn.1671-5411.2018.01.011, indexed in Pubmed: 29434622.

18. Alberti KG, Zimmet PZ Definition, diagnosis and classification of diabetes mellitus and its complications. Part 1: diagnosis and classification of diabetes mellitus provisional report of a WHO consultation. Diabet Med. 1998; 15(7): 539-553, doi: 10.1002/(SICI)1096-9136(199807)15:7<53 9::AID-DIA668 > 3.0.CO;2-S, indexed in Pubmed: 9686693.

19. Cobelli C, Toffolo GM, Dalla Man C, et al. Assessment of beta-cell function in humans, simultaneously with insulin sensitivity and hepatic extraction, from intravenous and oral glucose tests. Am J Physiol Endocrinol Metab. 2007; 293(1): E1-E15, doi: 10.1152/ajpendo.00421.2006, indexed in Pubmed: 17341552

20. Liu LL, Lawrence JM, Davis C, et al. SEARCH for Diabetes in Youth Study Group. Prevalence of overweight and obesity in youth with diabetes in USA: the SEARCH for Diabetes in Youth study. Pediatr Diabetes. 2010; 11(1): 4-11, doi: 10.1111/j.1399-5448.2009.00519.x, indexed in Pubmed: 19473302

21. Calanna S, Scicali R, Di Pino A, et al. Alpha- and beta-cell abnormalities in haemoglobin A1c-defined prediabetes and type 2 diabetes. Acta Diabetol. 2014; 51(4): 567-575, doi: 10.1007/s00592-014-0555-5, indexed in Pubmed: 24442427.

22. Shashaj B, Luciano R, Contoli B, et al. Reference ranges of HOMA-IR in normal-weight and obese young Caucasians. Acta Diabetol. 2016, 53(2): 251-260, doi: 10.1007/s00592-015-0782-4, indexed in Pubmed: 26070771.

23. Bi Y, Zeng L, Zhu D, et al. Association of -cell function and insulin sensitivity with fasting and 2-h plasma glucose in a large Chinese population. Diabetes Obes Metab. 2012; 14(2): 174-180, doi: 10.1111/j.1463-1326.201 1.01504.x, indexed in Pubmed: 21951345.

24. Mitsui R, Fukushima M, Nishi Y, et al. Factors responsible for deteriorating glucose tolerance in newly diagnosed type 2 diabetes in Japanese men. Metabolism. 2006; 55(1): 53-58, doi: 10.1016/j.metabol.2005.07.006, indexed in Pubmed: 16324919.

25. Sun M, Huang X, Jiang L, et al. Characterization of -cell function and insulin resistance in overweight Chinese adolescents with normal glucose tolerance. Exp Ther Med. 2013; 6(2): 547-551, doi: 10.3892/etm.2013.1164 indexed in Pubmed: 24137224

26. Aguayo-Mazzucato C. Functional changes in beta cells during ageing and senescence. Diabetologia. 2020; 63(10): 2022-2029 doi: 10.1007/s00125-020-05185-6, indexed in Pubmed: 32894312.

27. De Tata V. Age-related impairment of pancreatic Beta-cell function: pathophysiological and cellular mechanisms. Front Endocrinol (Lausanne). 2014; 5: 138, doi: 10.3389/fendo.2014.00138, indexed in Pubmed: 25232350.

28. Tramunt B, Smati S, Grandgeorge N, et al. Sex differences in metabolic regulation and diabetes susceptibility. Diabetologia. 2020; 63(3): 453-461, doi: 10.1007/s00125-019-05040-3, indexed in Pubmed: 31754750.

29. Kautzky-Willer A, Harreiter J, Pacini G. Sex and Gender Differences in Risk, Pathophysiology and Complications of Type 2 Diabetes Mellitus. Endocr Rev. 2016; 37(3): 278-316, doi: 10.1210/er.2015-1137, indexed in Pubmed: 27159875.

30. Khetan AK, Rajagopalan S. Prediabetes. Can J Cardiol. 2018; 34(5) 615-623, doi: 10.1016/j.cjca.2017.12.030, indexed in Pubmed: 29731022.

31. Defronzo RA. Banting Lecture. From the triumvirate to the ominous octet: a new paradigm for the treatment of type 2 diabetes mellitus. Diabetes. 2009; 58(4): 773-795, doi: 10.2337/db09-9028, indexed in Pubmed: 19336687

32. Cho JH, Kim JW, Shin JA, et al. -cell mass in people with type 2 diabetes. J Diabetes Investig. 2011; 2(1): 6-17, doi: 10.1111/j.2040-1124.2010 .00072.x, indexed in Pubmed: 24843456.

33. Zhang L, Qiao O, Tuomilehto J, et al. DECODE/DECODA Study Group. Distinct ethnic differences in lipid profiles across glucose categories. J Clin Endocrinol Metab. 2010; 95(4): 1793-1801, doi: 10.1210/jc.2009-2348, indexed in Pubmed: 20118302. 